INPLASY PROTOCOL

To cite: Wang et al. Safety and efficacy of hypoglycemic agents in patients with type 2 diabetes mellitus (T2DM): protocol for an overview of systematic reviews based on network meta-analysis. Inplasy protocol 202070118. doi: 10.37766/inplasy2020.7.0118

Received: 27 July 2020

Published: 27 July 2020

Corresponding author: Jing Liu

liuj996@126.com

Author Affiliation:

Department of Endocrinology,

Gansu Provincial Hospital, Lanzhou, China.

Support: None.

Review Stage at time of this submission: The review has not yet started.

Conflicts of interest:

The authors declared that there have no conflicts of interest to this work.

\section{Safety and efficacy of hypoglycemic agents in patients with type 2 diabetes mellitus (T2DM): protocol for an overview of systematic reviews based on network meta-analysis}

Wang, YF1; Xu, JG2; Quan, JX3; Niu, RL4; Hu, ZW5; Liu, J6.

Review question / Objective: For patients with type 2 diabetes mellitus (Participants), there are a variety of hypoglycemic agents (Interventions/ Comparators) to choose from (e.g. sulphonylureas, dipeptidyl peptidase 4 (DPP- 4) inhibitors, glucagon-like peptide 1 (GLP-1) analogues). Which type of agent has the best hypoglycemic efficacy and safety (Outcomes)?

Condition being studied: In 2011, the Ann Intern Med published the first network meta-analysis on the comparative effectiveness of glucose-lowering drugs for type 2 diabetes. In the following ten years, many similar NMAs appeared in this domain, comparing the safety and efficacy of different combination, dosage, course of treatment, and frequency of medication on type 2 diabetes between different hypoglycemic drugs. This overview will include a formal assessment of the methodological quality of included network meta-analysis and of the quality of evidence in included reviews.

INPLASY registration number: This protocol was registered with the International Platform of Registered Systematic Review and Meta-Analysis Protocols (INPLASY) on 27 July 2020 and was last updated on 27 July 2020 (registration number INPLASY202070118).

\section{INTRODUCTION}

Review question / Objective: For patients with type 2 diabetes mellitus (Participants), there are a variety of hypoglycemic agents (Interventions/ Comparators) to choose from (e.g. sulphonylureas, dipeptidyl peptidase 4 (DPP- 4) inhibitors, glucagonlike peptide 1 (GLP-1) analogues). Which type of agent has the best hypoglycemic efficacy and safety (Outcomes)? 
Rationale: Diabetes is a chronic and progressive disease characterized by a deterioration of blood glucose control over time. It is associated with significant clinical and economic burden globally.In 2000, the global estimate of adults living with diabetes was 151 million. A decade ago, by 2010 it had grown by $88 \%$ to 285 million, the global projection for diabetes in 2025 was 438 million.

Condition being studied: In 2011, the Ann Intern Med published the first network meta-analysis on the comparative effectiveness of glucose-lowering drugs for type 2 diabetes. In the following ten years, many similar NMAs appeared in this domain, comparing the safety and efficacy of different combination, dosage, course of treatment, and frequency of medication on type 2 diabetes between different hypoglycemic drugs. This overview will include a formal assessment of the methodological quality of included network meta-analysis and of the quality of evidence in included reviews.

\section{METHODS}

Search strategy: Our overview will search for systematic reviews from the following databases: Medline, Embase, Web of Science, PubMed and Cochrane Database of Systematic Reviews. Take pubmed as an example, the pre-search strategy is as follows: (("network meta analysis" OR "network meta analyses" OR "mixed treatment comparison meta analysis" OR "mixed treatment comparisons meta analyses" OR "mixed treatment meta analysis" OR "mixed treatment meta analyses" OR "mixed treatment comparisons" OR "mixed treatment comparison" OR "multiple treatment comparison meta analysis" OR "multiple treatment comparisons meta analyses" OR "multiple treatments meta analysis" OR "multiple treatments meta analyses" OR "multiple treatment meta analysis" OR "multiple treatment meta analyses" OR "multiple treatment comparison" OR "multiple treatment comparisons") AND diabetes).
Participant or population: We will limit our overview to studies of adults with T2DM regardless of gender or ethnic origins. The pregnant women with gestational diabetes will be excluded.

Intervention: Same as comparator. Comparisons among the following interventions were included: insulin, met formin, sulfonylureas, thiazolidinediones (TZDS), active comparator drugs (ACDs), dipeptidyl peptidase-4 (DPP-4) inhibitors, Glucagonlike peptide-1 (GLP-1) inhibitors, sodium/ glucose cotransporter 2 (SGLT-2) inhibitors, a-glucosidase inhibitors, glinides, liraglutide, dapagliflozin, rosiglitazone, meglitinides, or placebo. There are no combination formula restrictions on whether to plus other drugs to metformin or sulfonylureas. There are also no restrictions on the combination, dosage, and frequency between different drugs. We classify all eligible drugs according to the above drug categories and because different drugs in the same category may have a variable effect, we include studies that compare drugs in the same drug category either.

Comparator: Same as intervention. Comparisons among the following interventions were included: insulin, met formin, sulfonylureas, thiazolidinediones (TZDS), active comparator drugs (ACDs), dipeptidyl peptidase-4 (DPP-4) inhibitors, Glucagonlike peptide-1 (GLP-1) inhibitors, sodium/ glucose cotransporter 2 (SGLT-2) inhibitors, a-glucosidase inhibitors, glinides, liraglutide, dapagliflozin, rosiglitazone, meglitinides, or placebo. There are no combination formula restrictions on whether to plus other drugs to metformin or sulfonylureas. There are also no restrictions on the combination, dosage, and frequency between different drugs. We classify all eligible drugs according to the above drug categories and because different drugs in the same category may have a variable effect, we include studies that compare drugs in the same drug category either. 
Study designs to be included: We will include published, peer-reviewed network meta-analysis. Both direct comparison and indirect comparison of NMA will be included.

Eligibility criteria: We will include published, peer-reviewed network meta-analysis of drug therapy strategy for type 2 diabetes mellitus which provide meta-estimates for outcomes. Both direct comparison and indirect comparison of network metaanalysis will be included. We will limit our overview to studies of adults with T2DM regardless of gender or ethnic origins. The pregnant women with gestational diabetes will be excluded. Comparisons among the following interventions were included: insulin, metformin, sulfonylureas, thiazolidinediones (TZDs), active comparator drugs (ACDs), dipeptidyl peptidase-4 (DPP-4) inhibitors, Glucagonlike peptide-1 (GLP-1) inhibitors, sodium/ glucose cotransporter 2 (SGLT-2) inhibitors, a-glucosidase inhibitors, glinides, liraglutide, dapagliflozin, rosiglitazone, meglitinides, or placebo. There are no combination formula restrictions on whether to plus other drugs to metformin or sulfonylureas. There are also no restrictions on the combination, dosage, and frequency between different drugs. We classify all eligible drugs according to the above drug categories and because different drugs in the same category may have a variable effect, we include studies that compare drugs in the same drug category either. The primary outcomes are hemoglobin A1c (HbA1c) and fasting plasma glucose (FPG). There will be no restrictions by languages.

Information sources: Our overview will search for systematic reviews from the following databases: Medline, Embase, Web of Science, PubMed and Cochrane Database of Systematic Reviews. If any important data elements are missing or unclear and cannot be obtained from the relevant trials, we will contact the authors for information.

Main outcome(s): The primary outcomes are hemoglobin A1c (HbA1c) and fasting plasma glucose (FPG). The second outcomes are body mass index (BMI), $2 \mathrm{~h}$ postprandial blood glucose (2HPPG), body weight and adverse events, including hypoglycemia, diarrhea, upper respiratory tract infection (URTI), hypersensitivity reaction (HR), renal and hepatic toxicity.

Additional outcome(s): The second outcomes are body mass index (BMI), $2 \mathrm{~h}$ postprandial blood glucose (2HPPG), body weight and adverse events, including hypoglycemia, diarrhea, upper respiratory tract infection (URTI), hypersensitivity reaction (HR), renal and hepatic toxicity.

Data management: The retrieved articles from the databases were exported to EndNote X8 for duplicate removal and further categorization. The full text of reviews will also be uploaded and attached to EndNote X8. We shall perform predevelopment Microsoft Excel (version 16.16.5) spreadsheets to extract data and later export into tables and figures.

Quality assessment / Risk of bias analysis: Two reviewers will independently assess the methodological quality of included NMA using a revised version of the Assessment of Multiple Systematic Reviews-2 (AMSTAR-2) too. AMSTAR-2 was designed for systematic reviews of intervention studies. This tool consists of 14 items and is not meant to provide an overall score. Rather, authors will consider the effect of low scores for certain items on review quality and provide a high, moderate, low, or critically low overall confidence rating based on weaknesses in these critical domains. Any differences between author assessments will be resolved by discussion or adjudication by a third author. We will not exclude any reviews from the overview based on the results of this assessment.

Strategy of data synthesis: In our overview data summary tables, we will denote systematic reviews containing overlapping primary studies using appropriate footnotes; likewise, we will explicitly note systematic reviews removed from our evidence synthesis due to completely 
overlapping studies. We will discuss the potential impact of these exclusions when reporting the evidence synthesis.We will use forest plots to display published metaestimates for each agent and outcome. however, we will not compute an overview meta-estimate due to the likelihood of considerable heterogeneity in study populations and outcomes between studies, the absence of essential metadata and the lack of well-established quantification methods. We will present the findings as a narrative synthesis, and will take a visualized evidenced map. We will classify agents as having sufficient (green), probable (orange) or possible (red) evidence of safety and efficacy. We will consider evidence to be sufficient if a systematic review is of high quality.

Subgroup analysis: If there is a large heterogeneity among the included studies, we will not conduct subgroup analysis, but only make a narrative synthesis.

Sensibility analysis: If there is a large heterogeneity among the included studies, we will analyze the sources of heterogeneity from clinical and methodological perspectives, rather than sensibility analysis.

Language: There will be no restrictions by languages.

Country(ies) involved: All researchers were from China.

Keywords: Type 2 Diabetes Mellitus, Hypoglycemic Agents, overview of systematic reviews, network meta-analysis.

Dissemination plans: We will submit our findings for peer-review publication and presentation at national and international conferences. We will also disseminate our findings through established clinical networks, as well as consumer networks, using lay summaries where appropriate.

Contributions of each author:

Author 1 - Yunfang Wang.

Author 2 - Jianguo Xu.

Author 3 - Jinxing Quan.
Author 4 - Ruilan Niu.

Author 5 - Zhiwei Hu.

Author 6 - Jing Liu. 